\title{
ON COURNOT-NASH EQUILIBRIA IN GENERALIZED QUALITATIVE GAMES WITH AN ATOMLESS MEASURE SPACE OF AGENTS
}

\author{
M. ALI KHAN AND NIKOLAOS S. PAPAGEORGIOU
}

\begin{abstract}
We present a result on the existence of Cournot-Nash equilibria in games with an atomless measure space of players each with nonordered preferences and with strategy sets in a separable Banach space. Our result dispenses with any convexity assumption on the preference correspondence.
\end{abstract}

1. Introduction. Recent work of Kim, Prikry and Yannelis $[10,11]$, Yannelis [15], and the authors [8] has shown the existence of Cournot-Nash equilibria in games with a measure space of players, each of whose strategy sets lives in a separable Banach space and each of whose pay-offs is generated by a nonordered relation. This work solved a problem left open in [7] and relied on (previously unknown) selection theorems of the Carathéodory type. For motivation to the problem, the reader can see [6 and 8 ].

In this paper we present a result that dispenses with any kind of convexity assumption on the preference correspondence in games with an atomless measure space of players. Our result can be seen as an atomless analogue of the purely atomic result of Shafer and Sonnenschein [14], with the atomless hypothesis enabling us to dispense with their convexity-type assumption in spite of the infinitedimensional setting. Indeed, the theorem we present yields a new result even when it is specialized to a finite-dimensional Euclidean space (see Remark 2 below). As such, it generalizes the finite-dimensional results in Khan and Vohra [9]. Moreover, unlike $[\mathbf{8}, \mathbf{1 0}$, and 15] there is no need for elaborate selection theorems of the Carathéodory type. Our new method of proof is suggested by [4] in which Grodal asked whether the assumptions of Theorem 1 in Khan-Vohra [9] were consistent.

2. Preliminary definitions and the result. Let $(T, \Sigma, \mu)$ be a complete, finite measure space. Let $E$ denote a separable Banach space over the real numbers $R$ and $E^{*}$ its topological dual. The topology $\sigma\left(E, E^{*}\right)$ will be referred to as the weak topology on $E$. The norm in $E$ will be donoted by $\|\cdot\|$.

$P(E)$ will denote the hyperspace of all subsets of $E$ including the empty set $\{\varnothing\} . P_{f}(E)$ will denote the set of nonempty, closed subsets of $E$ and $P_{k}(E)$ the set of nonempty, compact subsets of $E$. A $w$ in front of $f$ or $k$ will mean that the

Received by the editors January 8, 1986 and, in revised form, April 18, 1986.

1980 Mathematics Subject Classification (1985 Revision). Primary 28A45, 90A14; Secondary 46G10, 90D13.

Key words and phrases. Measurable selector, multifunction, fixed point, generalized qualitative games, Cournot-Nash equilibrium.

We are indebted to an anonymous referee for his comments. Errors are solely ours. This research was supported in part by NSF grants to Brown University (Khan) and to the University of Illinois (Papageorgiou). 
closedness or compactness is with respect to the weak topology on $E$. A $c$ after $f$ or $k$ will denote that the set is in addition convex. For any $A, B$ in $P(E)$ we shall use the following notation.

con $A$ : convex hull of $A$.

$A^{c}$ : complement of $A$ in $E$.

$A \backslash B$ : set-theoretic subtraction.

We shall also denote the open $\delta$-ball around $x$ by $B_{\delta}(x)$ and the set of positive integers by $N$.

$L_{E}^{1}$ will denote the space of all (equivalence classes of) $E$-valued Bochner integrable functions defined on $T$ with

$$
\|f\|_{1}=\int_{T}\|f(t)\| d \mu .
$$

We shall abbreviate $L_{R}^{1}$ by $L^{1}$. For any multifunction $F: T \rightarrow P(E) \backslash\{\varnothing\}$, we shall denote by $S_{F}^{1}$ the set $\left\{f \in L_{E}^{1}: f(t) \in F(t)\right.$ a.e. in $\left.T\right\}$.

A multifunction $F: T \rightarrow P(E) \backslash\{\varnothing\}$ is said to be measurable if the graph of $F,\{(t, x) \in T \times E: x \in F(t)\}$, is an element of $\Sigma \otimes B(E)$ where $B(E)$ is the norm Borel $\sigma$-algebra on $E$ and $\otimes$ denotes the product $\sigma$-algebra. A measurable multifunction is said to be integrably bounded if there exists $g \in L^{1}$ such that $\sup \{\|x\|: x \in F(t)\} \leq g(t)$ a.e. in $T$.

Finally, for any two topological space $X, Y$, a multifunction $\psi: X \rightarrow P(Y) \backslash\{\varnothing\}$ is said to be upper hemicontinuous if the set $\{x \in X: \psi(x) \subset V\}$ is open in $X$ for every open subset $V$ of $Y$.

We now have all the terminology that we need to present our result. Following Schmeidler [13], we neglect the distinction between functions and their equivalence classes.

THEOREM. If

(1) $(T, \Sigma, \mu)$ is atomless and such that $L^{1}(\mu)$ is separable;

(2) $X: T \rightarrow P_{w k c}(E)$ is integrably bounded;

(3) $A: T \times S_{X}^{1} \rightarrow P_{w f c}(E)$ such that

(i) for all $t \in T$, for all $x \in S_{X}^{1}, A(t, x) \subset X(t)$,

(ii) for all $t \in T, A(t, \cdot)$ is an upper hemicontinuous multifunction from $\left(S_{X}^{1}\right.$, weak) to $(E$, norm $)$,

(iii) for all $x \in S_{X}^{1}$, the graph of $A(\cdot, x)$ belongs to $\Sigma \otimes B(E)$;

(4) $P: T \times S_{X}^{1} \rightarrow P(E) \backslash\{\varnothing\}$ such that

(i) for all $x \in S_{X}^{1}, x(t) \notin P(t, x)$ a.e. in $T$,

(ii) for all $t \in T, P^{c}(t, \cdot)$ is an upper hemicontinuous multifunction from $\left(S_{X}^{1}\right.$, weak) to $(E$, norm $)$,

(iii) for all $x \in S_{X}^{1}$, the graph of $P(\cdot, x)$ belongs to $\Sigma \otimes B(E)$;

then there exists $x^{*} \in S_{X}^{1}$ such that a.e. in $T, x^{*}(t) \in A\left(t, x^{*}\right)$ and $A\left(t, x^{*}\right) \cap$ $P\left(t, x^{*}\right)=\{\varnothing\}$.

REMARKS. 1. Note that our assumptions do not rule out the existence of $x \in S_{X}^{1}$ such that $P(t, x) \cap X(t)=\{\varnothing\}$ a.e. in $T$.

2. The result presented above is a far-reaching generalization of Theorem 2 in [9] for the case of an atomless measure space of players. It improves that result even when $E$ is assumed to be $R^{l}$ as can be seen by comparing the hypotheses on $A$. In 
particular, (4)(i) is a weakening of the hypothesis: for all $x \in S_{X}^{1}, x(t) \notin \operatorname{con} P(t, x)$ a.e. in $T$.

3. The result presented above relates to Theorem 2 in $[8]$ and Theorem 3.1 in [10]. From the viewpoint of potential applications in economics, it dispenses with the interiority hypothesis on $A(t, x)$ found in both papers. More generally, it allows one to dispense with the topology on $T$ and the Hausdorff continuity assumptions found in [8] and with the restrictive assumptions on $E$ found in [10]. However, neither Theorem 2 in [8] nor Theorem 3.1 in [10] require $\mu$ to be an atomless measure. This is no real restriction since the primary interest of this work is in showing the existence of pure strategy equilibria, in which case $\mu$ is atomless (see [7 and 13]).

3. Proof of the theorem. The novelty in the proof lies in Claim 4 and as remarked in the introduction, this claim is suggested by an assertion in [4]. The essential argument for the proof of this claim in the context of $R^{l}$ can be traced to [4].

Note that the proof makes no use of a utility indicator as in [9] or a Carathéodory selection theorem as in $[\mathbf{8}, \mathbf{1 0}$, and 15].

The proof consists of a series of claims.

ClAIM 1. $S_{X}^{1}$ is compact in the weak topology generated by the norm in $L_{E}^{1}$.

This is a consequence of a result of Papageorgiou [12, Theorem 4.2].

ClaIM 2. $S_{X}^{1}$ is nonempty and convex.

Convexity is a straightforward consequence of the convex valuedness of $X(\cdot)$. Nonemptiness is a consequence of the Aumann-St. Beuve measurable selection theorem [2, Theorem III.22].

ClaIM 3. The weak topology on $S_{X}^{1}$ is metrizable.

Since $E$ is a separable Banach space and $\mu$ is such that $L^{1}(\mu)$ is separable, $L_{E}^{1}$ is separable (see, for example, [5, p. 381]). The claim then follows as a consequence of the fact that weakly compact subsets of a separable Banach space are metrizable [3, Theorem V.6.3].

ClaIM 4. For all $x \in S_{X}^{1}, A(t, x) \cap P(t, x)=\{\varnothing\}$ a.e. in $T$.

If not, there exists $x^{\prime} \in S_{X}^{1}$ and $V \in \Sigma, \mu(V)>0$, such that

$$
A\left(t, x^{\prime}\right) \cap P\left(t, x^{\prime}\right) \neq\{\varnothing\} \quad \text { for all } t \in V .
$$

Denote the restriction of $\Sigma$ to $V$ by $\Sigma_{V}$ and the restriction of $\mu$ to $\Sigma_{V}$ by $\mu_{V}$. Since $A\left(\cdot, x^{\prime}\right)$ and $P\left(\cdot, x^{\prime}\right)$ have measurable graphs, the restrictions of $A\left(\cdot, x^{\prime}\right) P\left(\cdot, x^{\prime}\right)$ to $V$ also have measurable graphs. Hence the multifunction $A\left(\cdot, x^{\prime}\right) \cap P\left(\cdot, x^{\prime}\right)$ restricted to $V$ has a measurable graph. We can then appeal to the Aumann-St. Beuve selection theorem [2, Theorem III.22] to assert the existence of a measurable function $g: V \rightarrow$ $E$ such that

$$
g(t) \in A\left(t, x^{\prime}\right) \cap P\left(t, x^{\prime}\right) \quad \text { for all } t \in V .
$$

Since $A\left(t, x^{\prime}\right) \subset X(t)$ and $X(\cdot)$ is integrably bounded, pick $K \in R_{+}$such that $\mu(W)>\frac{2}{3} \mu(V)$ where

$$
W=\left\{t \in V:\|g(t)\|<K \text { and }\left\|x^{\prime}(t)\right\|<K\right\} .
$$

Next, for all $n$ in $N$, consider the set

$$
W_{n}=\left\{t \in W:\left\|x-x^{\prime}\right\|_{1}<1 / n \Rightarrow g(t) \in P(t, x)\right\} .
$$


We assert that for any $n \in N, W_{n} \in \Sigma_{V}$. To see this, note that the separability of $L_{E}^{1}$ implies that there exists a countable dense subset $\left\{x_{m}\right\}_{m \geq 1}$ of $B_{1 / n}\left(x^{\prime}\right)$. Now consider the set

$$
W_{n m}=\left\{t \in W: g(t) \in P\left(t, x_{m}\right)\right\} .
$$

Since $g$ is a measurable function, it has a measurable graph [2, III.36]. Since the restriction of $P\left(\cdot, x_{m}\right)$ to $V$ has a measurable graph, certainly the intersection of the graphs of $g$ and $P\left(\cdot, x_{m}\right)$ are measurable. The projection of this intersection on $V$ is the set

$$
V_{m}=\left\{t \in V: g(t) \in P\left(t, x_{m}\right)\right\} .
$$

By the projection theorem [2, Theorem III.23], $V_{m} \in \Sigma_{V}$. Since $W_{n m}=W \cap V_{m}$, $W_{n m} \in \Sigma_{V}$. Hence $\left(\bigcup_{m \geq 1} W_{n m}\right) \in \Sigma_{V}$. Denote this union by $W_{n}^{\prime}$. Certainly $W_{n}^{\prime} \subset W_{n}$. Finally, we show $W_{n} \subset W_{n}^{\prime}$. Towards this end, pick any $s \in W_{n}$. Then there exists $x_{s} \in B_{1 / n}\left(x^{\prime}\right)$ such that $g(s) \in P\left(s, x_{s}\right)$. Now by upper hemicontinuity of $P^{c}(t, \cdot)$, there exists $\delta(s)>0$ such that $g(s) \in P(s, x)$ for all $x \in B_{\delta(s)}\left(x_{s}\right)$. Certainly, there exists an element of $\left\{x_{m}\right\}_{m \geq 1}$, say $x_{m(s)}$, such that $x_{m(s)} \in B_{\delta(s)}\left(x_{s}\right)$. Hence $g(s) \in P\left(s, x_{m(s)}\right)$, and $s \in W_{n}^{\prime}$.

It is easy to see that $\bigcup_{n \geq 1} W_{n}=W$. Hence there exists $\bar{n}$ such that $\mu\left(W_{\bar{n}}\right)>$ $\frac{1}{2} \mu(V)$. Since $\mu$ is an atomless measure, we can pick $W^{*} \in \Sigma_{V}, W^{*} \subset W_{\bar{n}}$, such that $0<\mu\left(W^{*}\right)<1 / 2 K \bar{n}$. Define $x^{\prime \prime}: T \rightarrow E$ such that

$$
x^{\prime \prime}(t)= \begin{cases}g(t) & \text { for all } t \text { in } W^{*}, \\ x^{\prime}(t) & \text { for all } t \text { in } T \backslash W^{*}\end{cases}
$$

Certainly

$$
\left\|x^{\prime \prime}-x^{\prime}\right\|_{1}=\int_{W^{*}}\left\|g(t)-x^{\prime}(t)\right\| \leq \int_{W^{*}}\left(\|g(t)\|+\left\|x^{\prime}(t)\right\|\right)<2 K \mu\left(W^{*}\right)<1 / \bar{n} .
$$

This implies that $x^{\prime \prime}(t) \in P\left(t, x^{\prime \prime}\right)$ for all $t$ in $W^{*}$, a contradiction to (4)(i). The proof of our claim is complete.

Next, we construct the correspondence $\alpha: S_{X}^{1} \rightarrow S_{X}^{1}$ such that

$$
\alpha(x)=\left\{y \in S_{X}^{1}: y(t) \in A(t, x) \text { a.e. in } T\right\} .
$$

ClaIM 5. $\alpha$ is nonempty and convex valued.

Convex valuedness is obvious from the convexity of $A(t, x)$. Nonemptiness follows from an application of the Aumann-St. Beuve measurable selection theorem [2, Theorem III.22].

ClAIM 6. $\alpha$ is an upper hemicontinuous correspondence where $S_{X}^{1}$ is endowed with the weak topology.

The proof follows an argument in [8]; we spell it out here for the reader's convenience.

Since $S_{X}^{1}$ with the weak topology is a compact, metric space, it suffices to show that the graph of $\alpha$ is sequentially closed. Towards this end, let $y_{n} \in \alpha\left(x_{n}\right)$, $\left(y_{n}, x_{n}\right) \rightarrow(y, x)$. We have to show that $y \in \alpha(x)$. Let $A_{i}=\operatorname{con} \bigcup_{j \geq i} y_{j}$ for $i=$ $1,2, \ldots$ Since $y^{n}$ converges weakly to $y$, by Mazur's theorem [3, V.3.14], for each $i=1,2, \ldots$ there exists a sequence $\left\{z_{n}^{i}\right\}$ in $A_{i}$ such that $z_{n}^{i}$ converges strongly to $y$. For any $\varepsilon>0$, find $n_{1}$ such that $\left\|z_{n_{1}}^{1}-y\right\|<\varepsilon$. Similarly for $\varepsilon / m, m>1$, find $n_{m}$ such that $\left\|z_{n_{m}}^{m}-y\right\|<\varepsilon / m$. Proceeding in this way, we can manufacture a sequence, appropriately relabelled $\left\{z_{n}\right\}$, such that $z_{n} \in \operatorname{con} \bigcup_{j \geq n} y_{j}$ and $z_{n}$ converges in the 
$L_{E}^{1}$ norm to $y$. By passing to a subsequence, if necessary, we may assume that $z_{n}(t)$ converges in the $E$ norm to $y(t)$ for all $t \in T \backslash N$ with $\mu(N)=0$. Fix $t \in T \backslash N$. Since $A(t, \cdot)$ is upper hemicontinuous from $\left(S_{X}^{1}\right.$, weak) to $E$, for every $\delta>0$ we can find $n \geq 1$ such that $k \geq n$ implies $A\left(t, x_{k}\right) \subset A(t, x)+B_{\delta}(0)$. Hence

$$
\begin{aligned}
\operatorname{con} \bigcup_{k \geq n} A\left(t, x_{k}\right) & \subset A(t, x)+B_{\delta}(0) \\
& \Rightarrow z_{n}(t) \in A(t, x)+B_{\delta}(0) \\
& \Rightarrow y(t) \in A(t, x)+B_{\delta}(0) .
\end{aligned}
$$

On letting $\delta$ go to zero, we conclude that $y(t) \in A(t, x)$. Since $t \in T \backslash N$ was arbitrary, we also conclude that $y(t) \in A(t, x)$ a.e. in $T$. The proof of the claim is finished.

We can now apply Ky-Fan's fixed point theorem [1, §IX.5] to assert the existence of $x^{*} \in S_{X}^{1}$,

$$
x^{*}(t) \in A\left(t, x^{*}\right) \quad \text { a.e. in } T \text {. }
$$

By Claim 3, certainly,

$$
P\left(t, x^{*}\right) \cap a\left(t, x^{*}\right)=\varnothing \quad \text { a.e. in } T .
$$

The proof of the theorem is finished.

\section{REFERENCES}

1. C. Berge, Topological spaces, Macmillan, New York, 1963.

2. C. Castaing and M. Valadier, Convex analysis and measurable multifunctions, Lecture Notes in Math., vol. 580, Springer-Verlag, Berlin and New York, 1977.

3. N. Dunford and J. T. Schwartz, Linear operators, vol. 1, Wiley, New York, 1958.

4. B. Grodal, A comment on Khan-Vohra, private communication, September 1985.

5. A. Kolmogorov and S. Fomin, Introductory real analysis, Dover, New York, 1970.

6. M. Ali Khan, On extensions of the Cournot-Nash Theorem, Advances in Equilibrium Theory (C. D. Aliprantis et al., eds.), Springer-Verlag, Berlin and New York, 1985.

7. __ Equilibrium points of nonatomic games over a Banach space, Trans. Amer. Math. Soc. 293 (1986), 737-749

8. M. Ali Khan and N. Papageorgiou, On Cournot-Nash equilibria, Generalized Qualitative Games with a Continuum of Players, BEBR Faculty Paper No. 1126, March 1985; Nonlinear Anal. (to appear).

9. M. Ali Khan and R. Vohra, Equilibrium in abstract economies without ordered preferences and with a measure space of agents, J. Math. Econom. 13 (1984), 133-142.

10. T. Kim, K. Prikry, and N. Yannelis, Equilibria in abstract economies with a measure space and with an infinite dimensional strategy space, University of Minnesota Discussion Paper No. 218, July 1985.

11. _ On a Carathéodory-type selection theorem, Univ. of Minnesota Discussion Paper No. 217, July 1985.

12. N. S. Papageorgiou, Representation of set-valued operators, Trans. Amer. Math. Soc. 292 (1985), 557-572.

13. D. Schmeidler, Equilibrium points of nonatomic games, J. Statist. Phys. 7 (1973), 295-300.

14. W. Shafer and H. Sonnenschein, Equilibrium in abstract economies without ordered preferences, J. Math. Econom. 2 (1975), 345-348. 
15. N. C. Yannelis, Equilibria in non-cooperative models of competition, J. Econom. Theory (to appear).

16. N. C. Yannelis and N. D. Prabhakar, Existence of maximal elements and equilibria in linear topological spaces, J. Math. Ecomon. 12 (1983), 233-245.

DEPARTMENT OF ECONOMICS, UNIVERSity OF Illinois, 1206 South Sixth StREet, Champaign, Illinois 61820 (Current address of M. A. Khan)

Department of Mathematics, University of Illinois, 1409 W. Green Street, URBANA, ILLINOIS 61801

Current address (N. S. Papageorgiou): School of Technology, Division of Mathematical Sciences, University of Thessaloniki, Thessaloniki 504-06, Greece 